\title{
Tradução e adaptação transcultural da versão brasileira do Inventário de Ansiedade Geriátrica (GAl)
}

\section{Translation and cross-cultural adaptation of the Brazilian version of the Geriatric Anxiety Inventory (GAI)}

\author{
Camila Martiny ${ }^{1}$, Adriana Cardoso de Oliveira e Silva², Antonio Egidio Nardi³ ${ }^{3}$ Nancy Ann Pachana ${ }^{4}$
}

1 Psicóloga, Laboratório de Pânico e Respiração, Programa de Pós-Graduação, Instituto de Psiquiatria, Universidade Federal do Rio de Janeiro (UFRJ), Rio de Janeiro, RJ; INCT Translational Medicine.

${ }_{2}$ Pós-doutoranda do Programa de Pós-Graduação em Psiquiatria e Saúde Mental, Instituto de Psiquiatria/UFRJ; doutora em Psicologia, Instituto de Psicologia (IP), UFRJ; professora adjunta da Universidade Federal Fluminense (UFF); pesquisadora do Laboratório de Pânico e Respiração, Instituto de Psiquiatria, UFRJ; INCT Translational Medicine.

3 Livre-docente e professor-associado, Instituto de Psiquiatria, Faculdade de Medicina, Programa de Pós-Graduação em Psiquiatria e Saúde Mental, UFRJ; coordenador do Laboratório de Pânico e Respiração: INCT Translational Medicine.

${ }^{4}$ School of Psychology, University of Queensland, Brisbane, Austrália.

Recebido: 6/1/2010 - Aceito: 10/3/2010

\section{Resumo}

Introdução: É crescente a produção científica brasileira referente à adaptação de instrumentos utilizados internacionalmente para avaliação de ansiedade, porém a realização de estudos envolvendo ansiedade, especificamente na população geriátrica, ainda é menos frequente. Com isso, existe carência de instrumentos de medida criados com finalidade de atender essa população. A tradução e a adaptação transcultural são o primeiro passo para disponibilização de uma medida para investigação em diferentes contextos culturais, permitindo, desse modo, estudos comparativos. Objetivo: Elaborar a equivalência semântica da Geriatric Anxiety Inventory para o português brasileiro. Método: O processo foi realizado em quatro etapas: duas traduções; duas retrotraduções, ambas realizadas por tradutores cegos entre si e ao instrumento original; elaboração de uma versão sintética e pré-teste realizado com uma amostra da população-alvo. Resultados: Para cada item do instrumento, apresentam-se os resultados das quatro etapas. Ao longo do processo, eventuais problemas detectados puderam ser resolvidos, garantindo a qualidade do instrumento e sua adequação à população à qual se destina. Conclusão: A utilização do método em quatro etapas demonstrou ser eficaz e segura para o processo de equivalência semântica.

Martiny C, et al. / Rev Psiq Clín. 2011;38(1):8-12

Palavras-chave: Ansiedade, escala de mensuração, tradução.

\begin{abstract}
Introduction: There has been a growing interest in the cross-cultural adaptation of instruments for assessment of anxiety, but studies involving anxiety specifically in the geriatric population is still unusual. Thus, there is a lack of instruments designed to comply with this population. Translation and cultural adaptation is the first step to providing comparisons between different cultural contexts. Objective: Elaborate the semantic equivalence of the Geriatric Anxiety Inventory to Brazilian Portuguese. Method: The cross-cultural adaptation process consisted in four steps: two translations, two back- translations, both made by independent translators, the development of a synthetic version, and a pretest applied in a population sample. Results: For each item of the instrument, we present the results of four steps. Throughout the process, any problems found could be solved by ensuring the quality of the instrument and its appropriateness to the population to which it is intended. Discussion: The use of four-step method proved effective and safe in the process of semantic equivalence.
\end{abstract}

Martiny C, et al. / Rev Psiq Clín. 2011;38(1):8-12

Keywords: Anxiety, measure scale, translation.

\section{Introdução}

O Geriatric Anxiety Inventory (GAI) foi desenvolvido por Pachana et al1. como um instrumento breve para avaliação de ansiedade em população idosa. O inventário é composto por 20 itens dicotômicos em que o respondente deve marcar resposta declarando concordar ou discordar das afirmações apresentadas.

Sendo sua administração realizada de forma autoaplicada ou com aplicação individual, tanto oral quanto por meio de lápis e papel, permite que, mediante incapacidade do paciente para responder sozinho ao instrumento, a aplicação do GAI possa ser feita por um profissional de saúde.

Essa escala apresenta como vantagem a possibilidade de utilização com população que apresenta problemas de saúde mental, assim como com aquela que não o apresenta, sendo desse modo bastante amplo seu potencial de aplicação na prática. Internacionalmente, foi utilizada em diversos estudos ${ }^{4-8}$, apresentando bons resultados.

Outro ponto a ser destacado é que, enquanto escalas voltadas para avaliação de sinais e sintomas ligados à depressão em idosos são relativamente frequentes, havendo inclusive instrumento com esse propósito válido para a população brasileira ${ }^{2}$, os aspectos ligados à ansiedade ainda carecem de instrumentação adequada para sua avaliação ${ }^{3}$.

A possibilidade de avaliação rápida e eficaz é um forte ponto a favor do uso de escalas psicométricas, principalmente na área de saúde mental, onde ainda não se dispõem de marcadores biológicos ${ }^{9}$ que permitam a realização de diagnósticos com a mesma exatidão possível em outras áreas do conhecimento médico.

O baixo custo e a rapidez da apuração dos resultados também contribuem para o crescente investimento de profissionais de saúde 
mental nesse tipo de instrumentação. Nota-se nos últimos anos o interesse tanto no desenvolvimento de novas escalas para avaliação de ansiedade ${ }^{10-11}$ quanto na adaptação para o português brasileiro de instrumentos já utilizados internacionalmente ${ }^{12-20}$, o que apresenta ainda a vantagem de possibilitar comparações transculturais ${ }^{21}$ entre resultados obtidos por diferentes populações geográfica e culturalmente distantes.

Quanto aos seus parâmetros psicométricos, a GAI apresentou coeficiente alfa de Cronbach de 0,91 para população normal em idade avançada e 0,93 para população psicogeriátrica ${ }^{1}$. A validade concorrente do instrumento, analisada para os dois grupos, também se mostrou satisfatória e a aferição de fidedignidade por método de teste-reteste foi considerada excelente, desse modo apresentando também boa estabilidade temporal.

Os autores do instrumento original determinaram para sua população-alvo os valores 10/11 como ponto de corte para indicar a presença de ansiedade generalizada, conforme definição do DSM-IV-TR ${ }^{22}$.

Esse trabalho tem o objetivo de desenvolver a tradução e a adaptação semântica da GAI para o português brasileiro, incluindo aplicação experimental em uma amostra da população à qual ela se destina, com o objetivo de garantir a qualidade dessa adaptação. Esse trabalho possibilita futuros estudos de validação e aferição de fidedignidade dela para essa população específica.

\section{Métodos}

A metodologia adotada na obtenção da equivalência semântica é parte da adaptação transcultural, conforme proposto por Herdman et al. ${ }^{23}$ e empregada no Brasil por Reichenheim e Moraes ${ }^{24}$.

O processo utilizado na realização da adaptação transcultural da escala GAI foi composto por quatro etapas. As três primeiras etapas são: tradução, retrotradução e composição de uma versão sintética, baseada nas etapas anteriores. Após elas, é concluída a quarta etapa, que consiste na aplicação do instrumento como pré-teste na população-alvo.

$\mathrm{Na}$ primeira etapa, duas traduções do instrumento original, do inglês para o português, foram realizadas por dois tradutores bilíngues. Os tradutores trabalharam de forma independente e cega à escala original, mas tinham conhecimento técnico, por serem especialistas na área, a respeito dos termos utilizados no questionário.

$\mathrm{Na}$ segunda etapa, as duas traduções que foram feitas na primeira fase foram retrotraduzidas do português para o inglês por outros dois tradutores bilíngues, fluentes em inglês e também de forma independente e cega à versão original do instrumento. Os quatro tradutores que realizaram as duas traduções e das duas retrotraduções eram especialistas em saúde mental.

A terceira etapa consistiu da avaliação da equivalência semântica dos termos utilizados nas diferentes versões e sua comparação com os itens originais. Esta resultou na elaboração de uma versão sintética em português brasileiro, baseada nas duas traduções realizadas na primeira etapa.

Primeiramente, foi avaliada a equivalência da versão original do instrumento com as duas retrotraduções e, a seguir, cada item do instrumento original foi comparado com seu correspondente nas duas versões do português brasileiro. Para compor a versão sintética, alguns itens foram incorporados de uma das versões, integralmente ou modificados e também alguns itens foram criados da combinação das duas versões.

Após a elaboração da versão sintética, foi realizada a aplicação experimental do instrumento em 15 pessoas com idade entre 65 e 87 anos (média: 74,66; desvio-padrão: 40,54), sendo 9 indivíduos do sexo feminino e 6 indivíduos do sexo masculino. Estes foram agrupados em três categorias: escolaridade elementar (8 anos de estudo), escolaridade intermediária (11 anos de estudo) e ensino superior (aqueles que possuíam diploma universitário). Em cada categoria, participaram 5 indivíduos.

Todos os que foram selecionados para o pré-teste não apresentaram histórico de distúrbios mentais e foram submetidos à entrevista clínica estruturada para o DSM-IV (MINI, versão 5.0) ${ }^{25}$, com o intuito de eliminar qualquer possibilidade de diagnóstico psiquiátrico.

Os indivíduos que participaram do pré-teste assinaram um termo de consentimento livre e esclarecido concordando em fazer parte do estudo.

Todos os participantes foram solicitados a comentar sobre a versão sintética, apontando dificuldades e sugerindo adaptações que pudessem ser feitas com o objetivo de tornar o texto mais inteligível. Considerando tais sugestões e comentários, foi desenvolvida uma versão final do GAI para o português brasileiro.

\section{Resultados}

O instrumento original, as traduções (T1e T2), suas respectivas retrotraduções (B1 e B2) e a versão sintética estão demonstradas na tabela 1 .

As versões feitas pelos tradutores para os itens 2, 4, 6, 8, 10, 12, 17 e 18 foram idênticas ou muito semelhantes. Em alguns itens, uma versão teve prioridade sobre outra, ou ambas foram combinadas.

No primeiro item, "I worry a lot of the time", escolhemos a T1 "Eu me preocupo em grande parte do tempo", pelo fato de essa ser a tradução cujo sentido é o mais próximo do original, apesar de tanto em T1 como em T2 as retrotraduções B1 e B2 apresentarem diferenças na versão original. A T2 "Eu me preocupo muito o tempo todo" apresentou uma inexatidão na tradução, já que o tradutor inferiu algo que não foi dito na versão original, a palavra "muito" expressando ideia de quantidade.

No item 3, "I often feel jumpy", o termo "jumpy" pode ter variadas traduções como: saltitante, agitado, excitado, nervoso, inseguro e assustado. Apesar de tanto T1 como T2 apresentarem termos que correspondem à tradução correta da palavra jumpy, preferimos manter a tradução T1 "Sinto-me agitado com frequência", uma vez que o termo "agitado" tem o sentido mais próximo do original.

No item 5, "I often cannot enjoy things because of my worries", a tradução T1 "Frequentemente não consigo aproveitar as coisas por causa de minhas preocupações" foi escolhida por utilizar o termo "não consigo", dando similaridade à expressão original "cannot", ao passo que T2 omite a tradução desta.

O item 7 apresentou-se como um desafio por tratar-se de expressão idiomática. O termo "butterflies in my stomach" foi traduzido para o português brasileiro, em ambas as traduções (T1 e T2), como "borboletas no estômago". Tal expressão idiomática não existe em português brasileiro, logo teve que ser substituída por uma expressão equivalente. Com esse objetivo, utilizamos o termo "frio na barriga".

No item 9, a T2 apresentou uma inexatidão, já que a expressão "can't help" nesse contexto não tem o sentido de "não poder ajudar". A T1 "Não posso deixar de preocupar-me mesmo com coisas triviais" foi a única opção com equivalência semântica ao original.

No item 11, "My own thoughts often make me anxious", não houve diferenças significativas entre T1 e T2. A T2 "Meus próprios pensamentos com frequência me deixam ansioso" foi escolhida por causa de a retrotradução B2 feita a partir dela ser idêntica ao texto original.

No item 13, as traduções T1 e T2 foram idênticas, assim como as retrotraduções B1 e B2, mas preferimos fazer uma substituição do verbo "penso" pelo verbo "vejo" com o objetivo de tornar a frase mais coloquial e próxima da linguagem falada.

No item 14, "I always anticipate the worst will happen", optamos pela T1 "Eu sempre espero que o pior irá acontecer", pois essa tradução utilizou uma palavra similar à "antecipate" sem perder o sentido original. Apesar de "antecipate" em português brasileiro ser traduzido como "antecipar", a tradução literal não é uma forma muito utilizada e preferimos adotar a tradução contextualizada para que o entendimento do público-alvo seja mais claro.

No item 15, "I often feel shaky inside", tanto a palavra utilizada em T1, "tremendo", como em T2, "chacoalhando", foi empregada adequadamente como tradução de "shaky". Optamos pela T1, pois "tremendo" é mais utilizada e fácil de ser compreendida. 
Tabela 1. Versão original, traduções (T1 e T2), retrotraduções (R1 e R2) e versão sintética

\begin{tabular}{|c|c|c|c|c|}
\hline & Original & Tradução & Retrotradução & Versão sintética \\
\hline 1. & I worry a lot of the time & $\begin{array}{l}\text { T1: Eu me preocupo em grande parte do } \\
\text { tempo } \\
\text { T2: Eu me preocupo muito o tempo todo }\end{array}$ & $\begin{array}{l}\text { B1: I worry a great part of time } \\
\text { B2: I care too much all the time }\end{array}$ & $\begin{array}{l}\text { Eu me preocupo em grande parte } \\
\text { do tempo }\end{array}$ \\
\hline 2. & I find it difficult to make a decision & $\begin{array}{l}\text { T1: Eu acho difícil tomar uma decisão } \\
\text { T2: Eu acho difícil tomar uma decisão }\end{array}$ & $\begin{array}{l}\text { B1: I find it difficult to make a decision } \\
\text { B2: I find it difficult to make a decision }\end{array}$ & Eu acho difícil tomar uma decisão \\
\hline 3. & I often feel jumpy & $\begin{array}{l}\text { T1: Sinto-me agitado com frequência } \\
\text { T2: Sinto-me nervoso com frequência }\end{array}$ & $\begin{array}{l}\text { B1: I often feel agitated } \\
\text { B2: I often feel nervous }\end{array}$ & Sinto-me agitado com frequência \\
\hline 4. & I find it hard to relax & $\begin{array}{l}\text { T1: Acho difícil relaxar } \\
\text { T2: Eu acho difícil relaxar }\end{array}$ & $\begin{array}{l}\text { B1: I find it hard to relax } \\
\text { B2: I find it difficult to relax }\end{array}$ & Eu acho difícil relaxar \\
\hline 5. & $\begin{array}{l}\text { I often cannot enjoy things because } \\
\text { of my worries }\end{array}$ & $\begin{array}{l}\text { T1: Eu frequentemente não consigo } \\
\text { aproveitar as coisas por causa de minhas } \\
\text { preocupações } \\
\text { T2: Eu frequentemente não aproveito } \\
\text { as situações por causa das minhas } \\
\text { preocupações }\end{array}$ & $\begin{array}{l}\text { B1: I often can not enjoy things because } \\
\text { of my concerns } \\
\text { B2: I often do not take advantage of } \\
\text { situations because of my worries }\end{array}$ & $\begin{array}{l}\text { Eu frequentemente não consigo } \\
\text { aproveitar as coisas por causa de } \\
\text { minhas preocupações }\end{array}$ \\
\hline 6. & Little things bother me a lot & $\begin{array}{l}\text { T1: Pequenas coisas me aborrecem muito } \\
\text { T2: Pequenas coisas me aborrecem muito }\end{array}$ & $\begin{array}{l}\text { B1: Little things bother me a lot } \\
\text { B2: Little things bother me a lot }\end{array}$ & $\begin{array}{l}\text { Pequenas coisas me aborrecem } \\
\text { muito }\end{array}$ \\
\hline 7. & $\begin{array}{l}\text { I often feel like I have butterflies in } \\
\text { my stomach }\end{array}$ & $\begin{array}{l}\text { T1: Eu frequentemente sinto como se } \\
\text { tivesse borboletas no estômago } \\
\text { T2: Frequentemente eu me sinto como se } \\
\text { tivesse borboletas no meu estômago }\end{array}$ & $\begin{array}{l}\text { B1: Often feel like I have butterflies in } \\
\text { the stomach } \\
\text { B2: Often I feel like I have butterflies in } \\
\text { my stomach }\end{array}$ & $\begin{array}{l}\text { Eu frequentemente sinto como se } \\
\text { tivesse um "frio na barriga" }\end{array}$ \\
\hline 8. & I think of myself as a worrier & $\begin{array}{l}\text { T1: Eu penso em mim mesmo como } \\
\text { alguém preocupado } \\
\text { T2: Eu penso que sou preocupado }\end{array}$ & $\begin{array}{l}\text { B1: I think of myself as someone } \\
\text { concerned } \\
\text { B2: Eu penso que sou preocupado }\end{array}$ & Eu penso que sou preocupado \\
\hline 9. & $\begin{array}{l}\text { I can't help worrying about even } \\
\text { trivial things }\end{array}$ & $\begin{array}{l}\text { T1: Não posso deixar de preocupar-me } \\
\text { mesmo com coisas triviais } \\
\text { T2: Eu não posso ajudar preocupando-me } \\
\text { sempre com pequenas coisas }\end{array}$ & $\begin{array}{l}\text { B1: I can not help it to concern myself } \\
\text { even with trivial things } \\
\text { B2: I can not help worrying me always } \\
\text { with small things }\end{array}$ & $\begin{array}{l}\text { Não posso deixar de preocupar-me } \\
\text { mesmo com coisas triviais }\end{array}$ \\
\hline 10. & I often feel nervous & $\begin{array}{l}\text { T1: Frequentemente me sinto nervoso } \\
\text { T2: Frequentemente me sinto nervoso }\end{array}$ & $\begin{array}{l}\text { B1: I often feel nervous } \\
\text { B2: I often feel nervous }\end{array}$ & Frequentemente me sinto nervoso \\
\hline 11. & $\begin{array}{l}\text { My own thoughts often make me } \\
\text { anxious }\end{array}$ & $\begin{array}{l}\text { T1: Meus próprios pensamentos com } \\
\text { frequência me deixam ansioso } \\
\text { T2: Meus pensamentos me fazem sentir, } \\
\text { frequentemente, ansioso }\end{array}$ & $\begin{array}{l}\text { B1: My own thoughts often make me } \\
\text { anxious } \\
\text { B2: My thoughts often make me anxious }\end{array}$ & $\begin{array}{l}\text { Meus próprios pensamentos com } \\
\text { frequência me deixam ansioso }\end{array}$ \\
\hline 12. & $\begin{array}{l}\text { I get an upset stomach due to my } \\
\text { worrying }\end{array}$ & $\begin{array}{l}\text { T1: Tenho dor de estômago devido a minha } \\
\text { preocupação } \\
\text { T2: Tenho dores no estômago por causa } \\
\text { das minhas preocupações }\end{array}$ & $\begin{array}{l}\text { B1: I have stomach pain because of my } \\
\text { concern } \\
\text { B2: I have stomach pain because of my } \\
\text { worries }\end{array}$ & $\begin{array}{l}\text { Tenho dor de estômago por causa } \\
\text { das minhas preocupações }\end{array}$ \\
\hline 13. & $\begin{array}{l}\text { I think of myself as a nervous } \\
\text { person }\end{array}$ & $\begin{array}{l}\text { T1: Eu penso em mim mesmo como uma } \\
\text { pessoa nervosa } \\
\text { T2: Eu penso em mim como uma pessoa } \\
\text { nervosa }\end{array}$ & $\begin{array}{l}\text { B1: I think of myself as a nervous } \\
\text { person } \\
\text { B2: I think of myself as a nervous } \\
\text { person }\end{array}$ & $\begin{array}{l}\text { Eu me vejo como uma pessoa } \\
\text { nervosa }\end{array}$ \\
\hline 14. & $\begin{array}{l}\text { I always anticipate the worst will } \\
\text { happen }\end{array}$ & $\begin{array}{l}\text { T1: Eu sempre espero que o pior irá } \\
\text { acontecer } \\
\text { T2: Eu sempre antecipo que coisas ruins } \\
\text { vão acontecer }\end{array}$ & $\begin{array}{l}\text { B1: I always hope that the worst will } \\
\text { happen } \\
\text { B2: I always anticipate that bad things } \\
\text { will happen }\end{array}$ & $\begin{array}{l}\text { Eu sempre espero que o pior irá } \\
\text { acontecer }\end{array}$ \\
\hline 15. & I often feel shaky inside & $\begin{array}{l}\text { T1: Frequentemente me sinto tremendo } \\
\text { por dentro } \\
\text { T2: Eu frequentemente me sinto como se } \\
\text { estivesse chacoalhando por dentro }\end{array}$ & $\begin{array}{l}\text { B1: I often feel shivering inside } \\
\text { B2: I often feel like I'm shaking inside }\end{array}$ & $\begin{array}{l}\text { Frequentemente me sinto tremendo } \\
\text { por dentro }\end{array}$ \\
\hline 16. & $\begin{array}{l}\text { I think that my worries interfere } \\
\text { with my life }\end{array}$ & $\begin{array}{l}\text { T1: Eu acredito que minhas preocupações } \\
\text { interferem na minha vida } \\
\text { T2: Eu acho que minhas preocupações } \\
\text { interferem na minha vida }\end{array}$ & $\begin{array}{l}\text { B1: I believe that my concerns interfere } \\
\text { in my life } \\
\text { B2: I think my concerns interfere in } \\
\text { my life }\end{array}$ & $\begin{array}{l}\text { Eu acho que minhas preocupações } \\
\text { interferem na minha vida }\end{array}$ \\
\hline 17. & My worries often overwhelm me & $\begin{array}{l}\text { T1: Minhas preocupações me oprimem } \\
\text { com frequência } \\
\text { T2: Minhas preocupações frequentemente } \\
\text { me oprimem }\end{array}$ & $\begin{array}{l}\text { B1: My often worries overwhelm me } \\
\text { B2: My worries often overwhelm me }\end{array}$ & $\begin{array}{l}\text { Minhas preocupações } \\
\text { frequentemente me oprimem }\end{array}$ \\
\hline 18. & $\begin{array}{l}\text { I sometimes feel a great knot in my } \\
\text { stomach }\end{array}$ & $\begin{array}{l}\text { T1: Eu, algumas vezes, sinto um grande nó } \\
\text { em meu estômago } \\
\text { T2: Às vezes eu sinto como se tivesse um } \\
\text { grande nó no estômago }\end{array}$ & $\begin{array}{l}\text { B1: Sometimes I feel a big knot in my } \\
\text { stomach } \\
\text { B2: Sometimes I feel like a big knot in } \\
\text { my stomach }\end{array}$ & $\begin{array}{l}\text { Às vezes eu sinto como se tivesse um } \\
\text { grande nó no estômago }\end{array}$ \\
\hline 19. & $\begin{array}{l}\text { I miss out on things because I worry } \\
\text { too much }\end{array}$ & $\begin{array}{l}\text { T1: Eu perco coisas por me preocupar demais } \\
\text { T2: Eu erro e perco coisas porque me } \\
\text { preocupo demais }\end{array}$ & $\begin{array}{l}\text { B1: I lose things because I worry too much } \\
\text { B2: I commit mistakes and lose things } \\
\text { because I care too much }\end{array}$ & $\begin{array}{l}\text { Eu perco coisas por me preocupar } \\
\text { demais }\end{array}$ \\
\hline 20. & I often feel upset & $\begin{array}{l}\text { T1: Frequentemente me sinto aborrecido } \\
\text { T2: Frequentemente me sinto chateado }\end{array}$ & $\begin{array}{l}\text { B1: I often feel boring } \\
\text { B2: I often feel upset }\end{array}$ & Frequentemente me sinto chateado \\
\hline
\end{tabular}


No item 16, "I think that my worries interfere with my life", a T2 "Eu acho que minhas preocupações interferem na minha vida" foi escolhida, pois nesta a tradução de "I think" foi empregada de forma mais fidedigna ao original do que em T1. Em T1, a tradução desse termo foi "eu acredito", enquanto em T2 foi "eu acho". Tal diferença foi relevante para o entendimento da questão, após as retrotraduções. Para manter o sentido da versão original, optamos pela tradução T2.

No item 19, assim como no item 1, T2 apresentou inexatidão, pois o tradutor inferiu o termo "erro" que não foi mencionado no original, prejudicando a equivalência semântica. Assim sendo, elege-se T1 para compor a versão sintética.

No último item, tanto T1 como T2 apresentaram traduções análogas ao adjetivo "upset". Foi escolhido T2 por esta ter a retrotradução B2 mais similar ao original.

Em todos os casos, o pronome " $I$ ", em português traduzido por "Eu", foi excluído no início das frases, já que na linguagem coloquial do português brasileiro ele é pouco utilizado. Por meio da conjugação do verbo, pode-se inferir a pessoa do sujeito sem necessitar do uso de pronomes.

A quarta etapa, o pré-teste realizado com os participantes, tem como objetivo detectar possíveis equívocos e realizar a modificação de qualquer termo ou sentença que possa dificultar a compreensão do questionário. Os grupos de ensino elementar e intermediário realizaram poucas observações, não apresentaram dificuldades no entendimento e de maneira geral acharam o teste inteligível. Um indivíduo do grupo de ensino elementar relatou dificuldade no entendimento do item 5 por este ter um enunciado grande, o que o faria precisar ler mais de uma vez para entender a frase. Mas após três repetições da leitura, conseguiu compreender o significado. Optamos por não modificar o item 5 , pois a dificuldade apresentada não foi relacionada à semântica das palavras.

No grupo dos indivíduos que realizaram o ensino superior, dois sujeitos mencionaram grande repetição da palavra: "frequentemente". Tal observação não foi levada em conta, já que a noção de frequência é importante para o entendimento dos sintomas pelo profissional da saúde. Logo, esse termo foi mantido para não ocasionar prejuízo no diagnóstico.

Vale destacar que, dentre os 15 sujeitos participantes do pré-teste, havia dois de origem estrangeira: o primeiro indivíduo, de nacionalidade húngara, 87 anos de idade, sexo masculino e pertencente ao grupo dos indivíduos com ensino superior. Este relatou não ter tido dificuldades em compreender o significado dos 20 itens. $O$ segundo indivíduo, de nacionalidade portuguesa, 80 anos de idade, sexo feminino, e pertencente ao grupo dos indivíduos com ensino elementar, teve dificuldade em compreender o significado da palavra "triviais" e disse não conhecer tal expressão. Preferimos manter a palavra, pois todos os outros 14 participantes a conheciam. Os indivíduos estrangeiros moram no Brasil há 56 anos e 49 anos, respectivamente. Ambos demonstraram fluência na língua portuguesa brasileira e estão adaptados ao meio social e cultural do país, exercendo atividades cotidianas com facilidade.

\section{Discussão}

Para o uso de uma escala estrangeira, mantendo sua qualidade em outro contexto cultural, deve-se realizar um detalhado trabalho de tradução e adaptação. Sem tal, é possível que a medida perca confiabilidade por conta de diferenças linguísticas e culturais, que não são levadas em conta quando é realizada uma tradução simples ${ }^{26}$.

Com esse objetivo, enfatizamos na adaptação transcultural a equivalência semântica em detrimento da tradução literal dos termos, de acordo com as diretrizes propostas na literatura ${ }^{14}$.

A adaptação de uma escala já existente pode ter diversas vantagens, como fornecer uma medida-padrão para investigação em diferentes contextos culturais, o que possibilitaria a realização de estudos internacionais mediante comparações de um mesmo fenômeno em diversas culturas ${ }^{27}$.

Este estudo adotou a metodologia de adaptação transcultural por três etapas e um pré-teste. A criação de uma versão sintética composta a partir de duas traduções e duas retrotraduções propiciou uma meticulosa análise em que pudemos identificar discrepâncias e alcançar, assim, a melhor adaptação possível.

Apesar das dificuldades em adaptar certas expressões idiomáticas que são inexistentes na língua portuguesa brasileira, como o caso de "butterflies on stomach", pudemos solucionar essa questão substituindo por uma expressão de valor semântico equivalente, sem perder o sentido do original.

$\mathrm{Na}$ tradução, podem ser adicionadas palavras que foram inferidas pelo tradutor e que não constavam no texto original, por isso se destaca a importância do método utilizado, evitando determinadas distorções do original.

A aplicação do pré-teste foi útil para comprovação de que a versão final estava acessível para todos os graus de escolaridade. As sugestões feitas pelos participantes foram discutidas com o intuito de chegar a uma versão final mais apropriada à população-alvo.

Apesar de termos um pequeno número de sujeitos $(\mathrm{n}=15)$, aplicamos em três grupos homogêneos quanto ao tamanho e heterogêneo em relação a outras características como idade e escolaridade, o que possibilitou uma avaliação da viabilidade de aplicação dessa escala na população idosa brasileira.

Um ponto de destaque neste estudo foi a participação de dois indivíduos estrangeiros, porém totalmente ambientados na cultura da população-alvo no pré-teste, demonstrando a aplicabilidade do teste em indivíduos de outras nacionalidades adaptados à cultura brasileira.

A adaptação transcultural e a equivalência semântica são apenas a primeira fase para a validação de um instrumento de medida. É importante que o instrumento tenha boa confiabilidade e validade ${ }^{13}$.

Sugerem-se futuros estudos de suas propriedades psicométricas, de modo que a GAI possa ser adotada em trabalhos de pesquisa que estudem aspectos ligados à ansiedade na população à qual ela se destina, ou mesmo ser incluída na prática clínica, preenchendo uma importante lacuna nesse campo de trabalho.

\section{Nome da agência de fomento que concedeu auxílio}

Conselho Nacional de Desenvolvimento Científico e Tecnológico (CNPq).

\section{Referências}

1. Pachana NA, Byrne GJ, Siddle H, Koloski N, Harley E, Arnold E. Development and validation of the Geriatric Anxiety Inventory. Int Psychogeriatr. 2007;19(1):103-14.

2. Paradela EMP, Lourenço RA, Veras RP. Validação da escala de depressão geriátrica em um ambulatório geral. Rev Saude Publica. 2005;39:918-23.

3. Byrne JAG. What happens to anxiety disorders in later life? Rev Bras Psiquiatr. 2002;24:74-80.

4. Cully JA, Jimenez DE, Ledoux TA, Deswal A. Recognition and treatment of depression and anxiety symptoms in heart failure. Prim Care Companion J Clin Psychiatry. 2009;11(3):103-9.

5. Diefenbach GJ, Tolin DF, Meunier SA, Gilliam CM. Assessment of anxiety in older home care recipients. Gerontologist. 2009;49(2):141-53.

6. Boddice G, Pachana NA, Byrne GJ. The clinical utility of the geriatric anxiety inventory in older adults with cognitive impairment. Nurs Older People. 2008;20(8):36-9.

7. Rozzini L, Chilovi BV, Peli M, Conti M, Rozzini R, Trabucchi M, et al. Anxiety symptoms in mild cognitive impairment. Int J Geriatr Psychiatry. 2009;24(3):300-5.

8. Andrew DH, Dulin PL.The relationship between self-reported health and mental health problems among older adults in New Zealand: experiential avoidance as a moderator. Aging Ment Health. 2007;11(5):596-603.

9. Versiani M. Princípios gerais básicos das escalas de avaliação. In: Centro de Pesquisa em Psicobiologia Clínica, editor. Escalas de avaliação para monitorização de tratamentos com psicofármacos. São Paulo, Associação Fundo de Incentivo à Psicofarmacologia; 1989.

10. Del Prette ZAP, Del Prette A. Inventário de habilidades sociais. São Paulo: Casa do Psicólogo; 2001.

11. Hultz CS, Nunes CHSS. Escala Fatorial de Socialização. São Paulo: Casa do Psicólogo; 2007. 
12. Levitan MN, Nascimento I, Freire RC, Mezzasalma MA, Nardi AE. Equivalência semântica da versão brasileira da Social Avoidance and Distress Scale (SADS). Rev Psiquiatr Rio Gd Sul. 2008;30:49-58.

13. Berger W, Mendlowicz MV, Souza WF, Figueira I. Equivalência semântica da versão em português da Post-Traumatic Stress Disorder Checklist-Civilian Version (PCL-C) para rastreamento do transtorno de estresse pós-traumático. Rev Psiquiatr Rio Gd Sul. 2004;26:167-5.

14. Vilete L, Figueira I, Coutinho E. Adaptação transcultural para o português do Social Phobia Inventory (SPIN) para utilização entre estudantes adolescentes. Rev Psiquiatr Rio Gd Sul. 2006;28:40-8.

15. Scagliusi FB, Cordás TA, Polacow VO, Coelho D, Alvarenga M, Philippi ST, et al. Tradução da escala de desejo de aceitação social de Marlowe \& Crowne para a língua portuguesa. Rev Psiquiatr Clín. 2004;31:272-8.

16. Picon P, Gauer GJC, Fachel JMG, Manfro GG. Desenvolvimento da versão em português do Social Phobia and Anxiety Inventory (SPAI). Rev Psiquiat Rio Gd Sul. 2005;27:40-50.

17. Silva ACO, Nardi AE. Tradução e adaptação transcultural da versão brasileira da Fear of Negative Evaluation Scale (FNE). Rev Psiquiatr Rio Gd Sul. [In press].

18. Silva ACO, Nardi AE. Social Interaction Self-Statement Test (SISST): tradução e adaptação transcultural. Rev Psiquiatr Clín. 2010;37(5):199-205.

19. Sardinha A, Nardi AE, Eifert GH. Tradução e adaptação transcultural da versão brasileira do Questionário de Ansiedade Cardíaca. Rev Psiquiatr Rio Gd Sul. 2008;30:1-10.
20. Sardinha A, Levitan MN, Lopes FL, Perna G, Esquivel G, Griez EJ, et al. Tradução e adaptação transcultural do questionário de atividade física habitual. Rev Psiquiatr Clín. 2010;37(1):16-22.

21. Lima LA, Gonçalves S, Lovisi G, Pereira BB. Validação transcultural da Escala de Avaliação de Limitações do Comportamento Social - SBS-BR. Rev Psiquiatr Clín. 2003;30:126-38.

22. Associação Psiquiátrica Americana (APA). DSM-IV-TR. Manual diagnóstico e estatístico de transtornos mentais: DSM-IV-TR. Porto Alegre: Artmed; 2003.

23. Herdman M, Fox-Rushby J, Badia X. "Equivalence" and the translation and adaptation of the health-related quality of life questionnaires. Qual Life Res. 1997;6(3):237-47.

24. Reichenheim ME, Moraes CL. Operacionalização de adaptação transcultural de instrumentos de aferição usados em epidemiologia. Rev Saude Publica. 2007;41(4):665-73.

25. Sheehan DV, Lecrubier Y, Sheehan KH, Amorim P, Janavs J, Weiller E, et al. The Mini International Neuropsychiatric Interview (MINI): the development and validation of a structured diagnostic psychiatric interview for DSM-IV and ICD-10. J Clin Psychiatry. 1998;59(Suppl. 20):22-33.

26. Berkanovic $\mathrm{E}$. The effect of inadequate language translation on Hispanics' responses to health surveys. Am J Public Health. 1980;70:1273-6.

27. Guillemin F. Cross-cultural adaption and validation of the health status measures. Scand J Rheumatol. 1995;24:61-3. 